\title{
Detecting filter-cake pathologies in solid-liquid filtration: semi-tech scale demonstrations using electrical resistance tomography (ERT)
}

\author{
D. Vlaev ${ }^{\mathrm{a}, *}$, M. Wang ${ }^{\mathrm{a}}$, T. Dyakowski ${ }^{\mathrm{a}}$, R. Mann ${ }^{\mathrm{a}}$, B.D. Grieve ${ }^{\mathrm{b}}$ \\ a Virtual Centre for Industrial Process Tomography, Department of Chemical Engineering, UMIST, Manchester M60 1QD, UK \\ ${ }^{\mathrm{b}}$ Virtual Centre for Industrial Process Tomography, Zeneca, Huddersfield Works, Leeds Road, Huddersfield, UK
}

\begin{abstract}
Solid-liquid filtration monitoring by means of a single inexpensive 16-element ring sensor array for electrical resistance tomography (ERT) is described. The high sensitivity of the UMIST Mark 1b-E data acquisition system led to the unexpected finding that this tomography array can detect movement of the liquid level during filtration. This degree of sensitivity was also capable of detecting any tilt of the filter assembly, so that pathological behaviour due to malfunction or accidental displacement of the filter support plate could also be identified. Moreover, illustrative distortions of a filter cake formed by artificial surface depressions were readily observed in tomograms reconstructed by linear back projection. It is concluded that ERT has great potential for instrumenting and detecting 'pathological' behaviour of filtration processes in the pharmaceutical and fine chemicals industries. (C2000 Elsevier Science S.A. All rights reserved.
\end{abstract}

Keywords: Filtration; Resistance tomography; Process pathology

\section{Introduction}

Process tomography applications in industry are widening as the medical concept of non-invasive body-scanning is increasingly developed for deployment on chemical process units [1,2]. Electrical techniques developed and applied at UMIST encompass electro-magnetic [3], capacitance [4] and resistance [5] tomography. Some examples of applications to chemical processes include:

temperature profiles [6]

drainage of porous media [7]

two-phase pipe flow [8]

fluidised beds [4]

three phase trickle-beds [9]

stirred vessel mixing $[5,10]$.

This paper describes an application of electrical resistance tomography (ERT) to a typical solid-liquid filtering process.

\section{Tomography sensor system for a filter}

Fig. 1 shows a schematic view of a $1 \mathrm{~m}$ diameter semi-tech scale filter. These typically consist of a perforated support plate upon which is mounted a suitable cloth. When mother liquor and associated suspended solid are charged into the filter, the solid material settles out and is retained on the cloth

\footnotetext{
* Corresponding author.
}

where it accumulates, eventually forming a relatively thick cake. The mother liquor simultaneously flows first through the cloth and subsequently through thickening cake as it is drawn off from the base. It is usually important that the cake forms uniformly as the mother liquor drains through it. This ensures an even distribution of voidage in the cake within the voids of which some mother liquor will inevitably be retained. Any subsequent washing of the cake with a wash liquor should then preferably give rise to uniform rinsing so that any traces of mother liquor are effectively and efficiently displaced. In this way, drying of the final cake should not leave unevenly contaminated patches of solid. The uniformity of washing and drying are also crucially dependent on the uniform formation and lying-down of the filter cake onto the filter cloth.

Such conventional filter configurations (often operated under pressure) usually possess little by way of instrumentation for monitoring and safeguarding the evenness and uniformity of the solid cake. This is despite the fact that in the fine chemicals and pharmaceutical industries, the solid products produced by filtration after batch reaction may be of very high value. Consequently, any inappropriate contamination can drastically reduce the potential value of the product for which high purity is usually mandatory.

In order to provide the potential for improved monitoring of filter cake formation and cleansing, this paper reports on the deployment of a simple and inexpensive ring of ERT sensing plates as indicated in Fig. 1. This comprises 16 


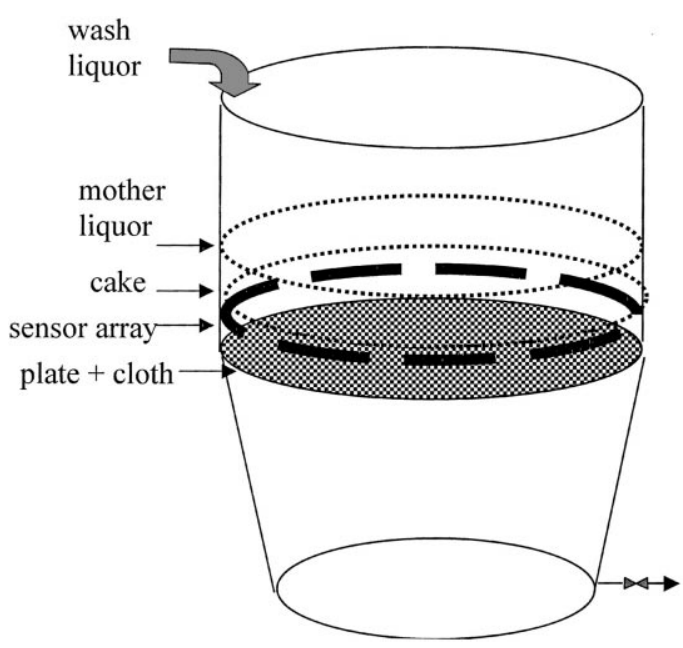

Fig. 1. UMIST test filter.

stainless steel sensing plates designed to be operated using an adjacent pair protocol by the UMIST Mark 1b-E data acquisition system (DAS). Each plate has dimensions of $66 \times 25 \mathrm{~mm}$ and they are equally spaced around the filter (inner) circumference forming a ring $10 \mathrm{~cm}$ above the support plate. The plates are positioned and held in a polypropylene trunking which houses and leads-off the wires connecting each plate to the DAS. Fig. 2 shows an overhead view of the sensor ring in position. A vertical trunking is used to connect the collected leads and pass them from the filter body to the DAS. The filter cloth can be seen in place in Fig. 2 and the

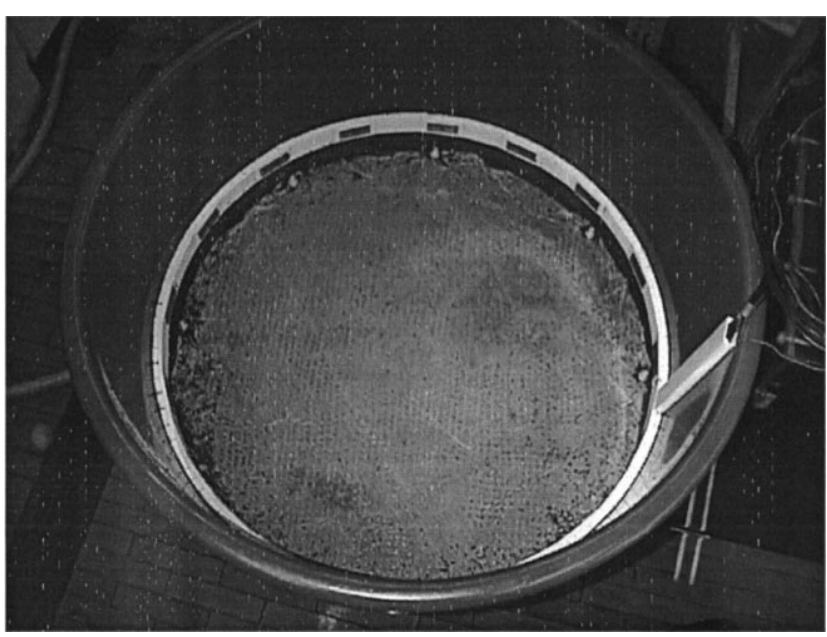

Fig. 2. Overhead view of the test filter with electrode array in position.

drilled holes in the support plate (which allow passage of the filter liquids) are partly visible through it. Three aspects of filter monitoring were investigated with this pilot test unit:

(i) monitoring of liquid level

(ii) detection of small degrees of tilt in the filter assembly

(iii) detection of induced artefact malformations in a wet cake of solid.

The second and third aspects shows how 'pathological' features in the filtering process can in principle be sensitively identified by ERT.

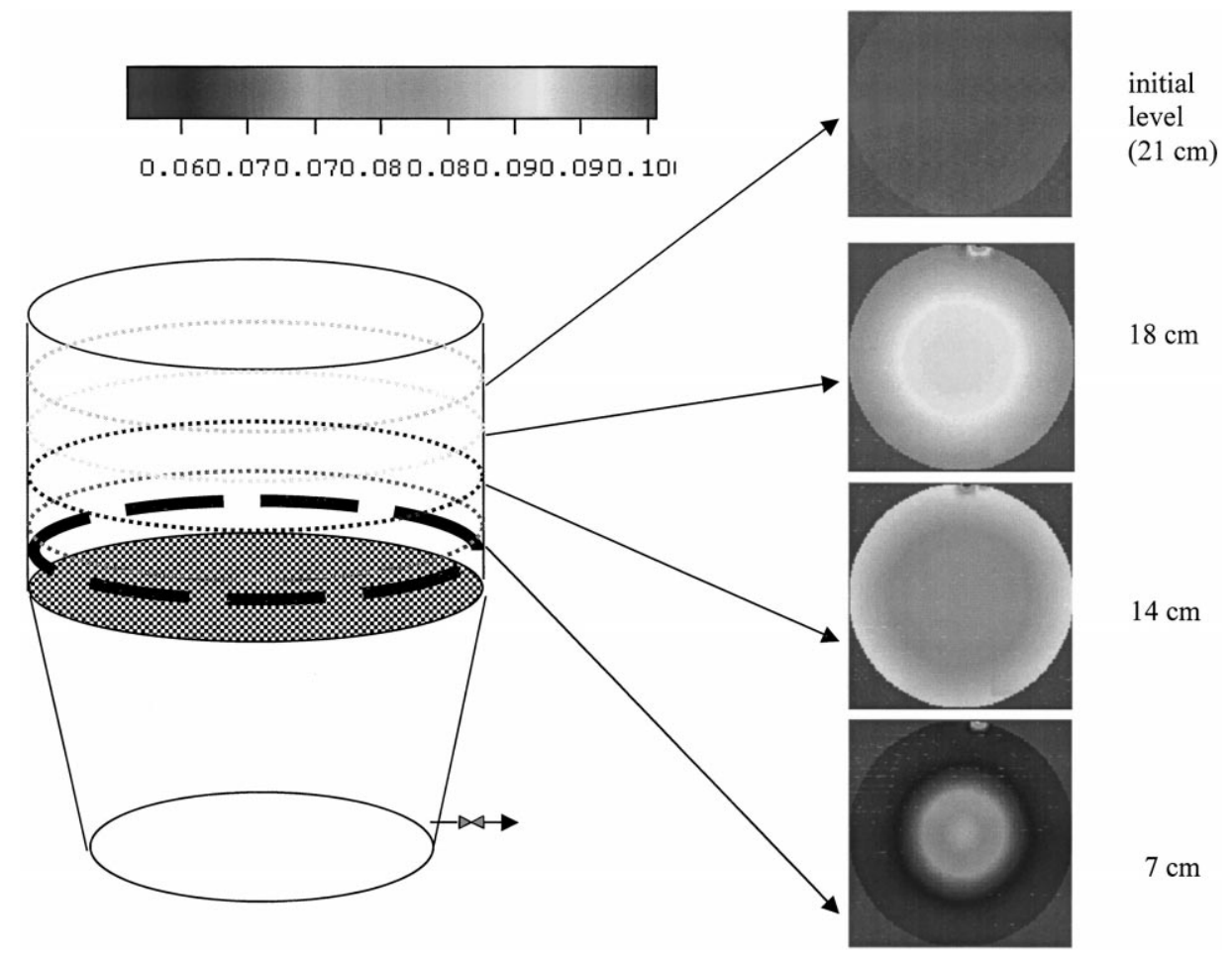

Fig. 3. Mother liquor level detection during filtration using ERT. 


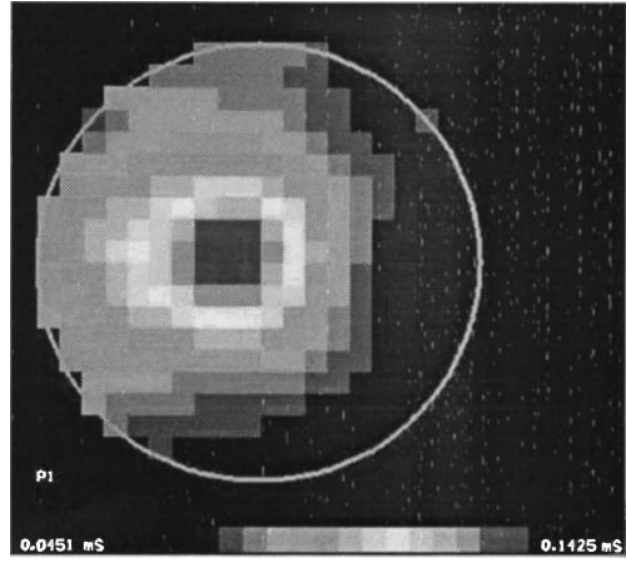

Fig. 4. Tilt detection using ERT.

\section{Level detection during liquor drainage}

The circumferential ERT sensing array is non-invasive and proved to be capable of remarkable sensitivity in detecting changes in conductivity/resistivity. Unexpectedly, preliminary tests showed clearly that the array could measure resistivity changes accompanying the fall in liquor level above the filter cake and plate. The results are illustrated in Fig. 3. Using an initial zeroing of the sensed resistively at a starting level of $21 \mathrm{~cm}$ above the support plate, the set of resistivities measured and reconstructed by linear back projection produced the tomogram images shown in Fig. 3 for liquor heights of 18,14 and $7 \mathrm{~cm}$. These cross section views reflect the fact that the resistivity field in 3-D changes as the level of (conducting) fluid descends towards the support plate, so that the filter sensing array becomes a potential liquid level detector. This capability could be extremely useful in monitoring the progress of filtration. The reconstructed sections are not uniform, although they are symmetrical. The non-uniformity is thought to be an artefact caused by the changing 3-D geometry of the resistivity field interacting with the reconstruction technique. Thus, some care will be needed in interpreting this effect and additional calibrations will be needed to read-off the actual level in real-time plant operation. Nevertheless, the use of such an ERT array to follow the flow of filtrate/mother liquor presents a new opportunity to improve filtration instrumentation.

\section{Tilt detection during drainage}

The sensitivity of the ERT array to small changes in local conductivity also provided another unanticipated measurement possibility. If at some point, the peripheral sensing array is displaced from the horizontal, the liquid level remains perfectly horizontal but the sensing protocol can detect an imbalance in the set of tomographic measurements. Upon reconstruction by linear back projection, the image can show a clear loss of symmetry as shown in Fig. 4. This sensitivity to tilt could offer a practical possibility for detecting pathologies associated either with initially improper assembly of the support plate/sensor array or alternatively a subsequent accidental displacement or malfunction following a proper initial installation. The tomography sensor array can thus provide potential information on correct assembly and on-going mechanical integrity of a filter system.

\section{Malformation in a wet cake}

The original purpose in developing ERT to monitor process filters was to see whether the Mark 1b-E DAS could detect malformations or so-called 'pathologies' in a forming filter cake. In light of the above demonstrations of the extreme sensitivity of the system, it was not surprising that cake distortions could be readily imaged in the reconstructed tomograms. Some initial results are presented in Figs. 5 and 6.

Fig. 5 shows a view of two small depressions formed next to each other near the filter wall. These were crudely
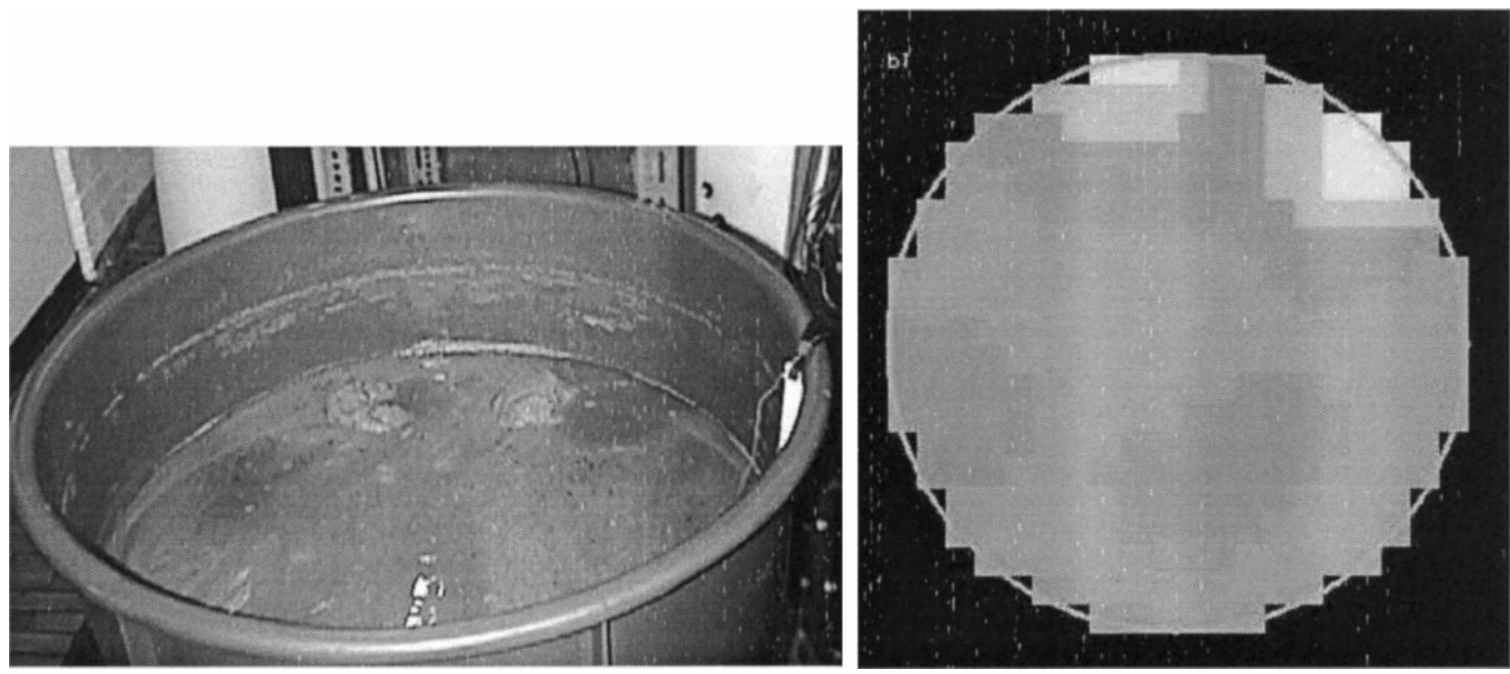

Fig. 5. On-line detection of two shallow depressions in a sand filter cake. 

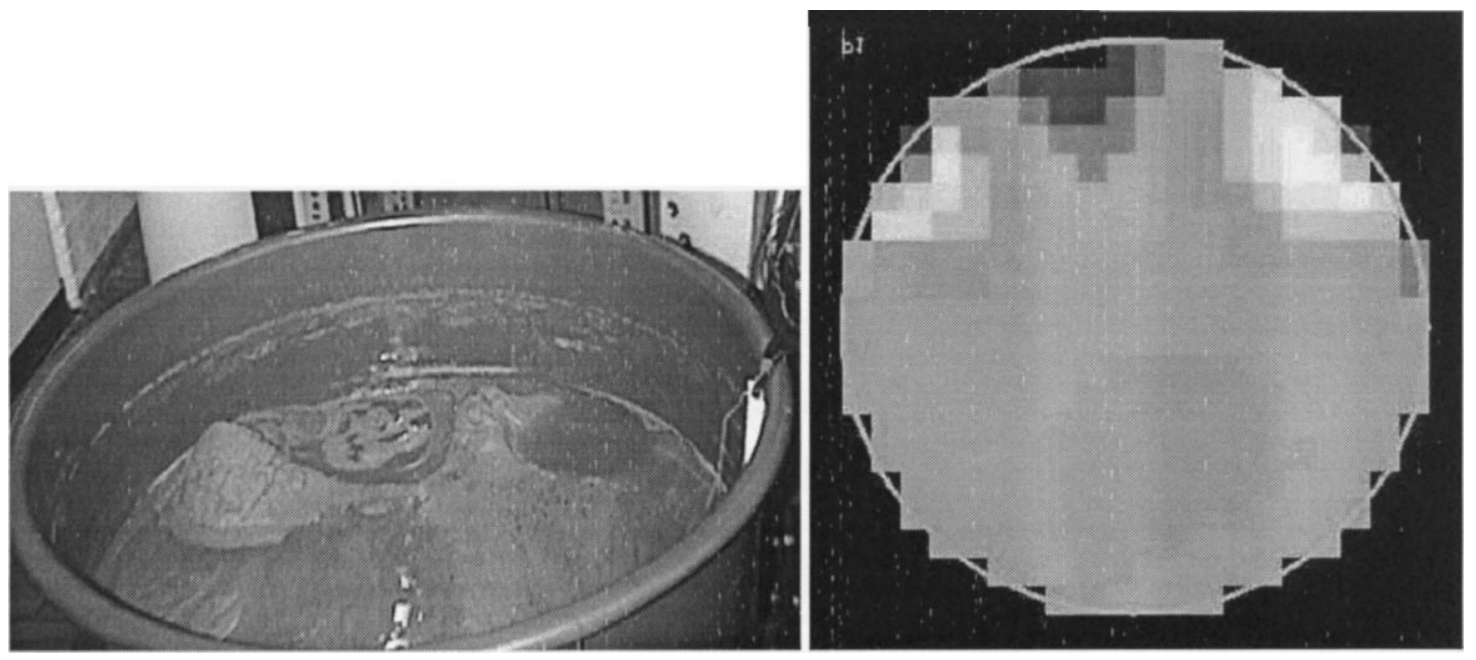

Fig. 6. On-line detection of two large depressions in a sand filter cake.

formed by hand giving approximately circular holes of shallow depth (about $3 \mathrm{~cm}$ ) and about $10 \mathrm{~cm}$ in diameter. These small indentations can be clearly seen in the reconstructed image based on a maximum/minimum range of conductivity from 0.083 to $0.118 \mathrm{mS} \mathrm{cm}^{-1}$.

Fig. 6 then shows the result for deeper and larger depressions. In this figure, the depressions are roughly twice the size of those in Fig. 5, being about $5 \mathrm{~cm}$ deep and $15 \mathrm{~cm}$ across. This pair of artefacts in the cake structure show up much more intensively in Fig. 6, using the same range of conductivity. The removal of solid from a section of the wet cake leaves the resulting 'hole' full of filtrate liquor, so these depressions show up as local regions of higher conductivity.

These preliminary results confirm that issues of cake non-uniformity, malformation, and unevenness are capable of detection from tomograms created by linear back-projection from a simple single-plane 16-element sensor array.

\section{Conclusions}

It is concluded that a single inexpensive array of ERT sensors provides the capability for detailed monitoring of a typical filtration process. The high sensitivity of the UMIST Mark 1b-E data acquisition system has shown an unanticipated capability for both filtrate level monitoring and tilt detection associated with mis-assembly or malfunction. Good sensitivity to minor imperfections in the cake has been demonstrated by virtue of the system's ability to detect and distinguish pairs of small and large indentations artificially created in a fully wetted cake.

Additional electrode ring(s) (at a different level) could allow a clear distinction to be made between level changes or tilt and pathological anomalies when they occur simultaneously. Further advances in tomography, such as multimodality data acquisition could also be used for this purpose.

Further possibilities exist for additional capabilities including the important issue of monitoring washing efficiency and the identification of dryness induced by pressure filtration forcing an air flow through the cake. Also, cake non-uniformities induced by other malfunctions, such as uneven filtrate flow, if the cloth is torn or the support plate holes are partly blocked, should also be detectable by ERT.

\section{Acknowledgements}

The authors gratefully acknowledge support by EPSRC under the Foresight Challenge Programme, Grant GR/L 37434 .

\section{References}

[1] R.A Williams, M.S. Beck (Eds.), Process Tomography-Principles, Techniques and Applications, Butterworth-Heinemann, Oxford, 1995

[2] J. Chaouki., F. Lavachi, M.P. Dudokovics, Non-Invasive Monitoring of Multi-Phase Flows, Elsevier, New York, 1997

[3] Z.Z. Yu., A.J. Peyton, M.S. Beck, W.F. Conway, L.A. Xu, Imaging Systems Based on Electromagnetic Tomography, Electronic Lett. 29(7) (1993) 625-626.

[4] T. Dyakowski, R.B. Edwards, C.G. Xie, R.A. Williams, Application of Capacitance Tomography to Gas-Solid Flows, Chem. Eng. Sci. 52 (1997) 2099-2110.

[5] R. Mann, F.J. Dickin, M. Wang, T. Dyakowski, R.A. Williams, R.B. Edwards, A.E. Forrest, P.J. Holden, Application of Electrical Resistance Tomography to Interrogate Mixing Processes at Plant Scale, Chem. Eng. Sci. 52 (13) (1997) 2087-2097.

[6] W. Ostendorf, D. Mewes, F. Mayinger, A tomographical method using holographic interferometry for the registration of 3-D unsteady temperature profiles in laminar and turbulent flow, in: C.L. Tiens (Ed.), Heat-Transfer 1986, Proc. 8th Int. Heat Transfer Conf., Vol. 2, Hemisphere, New York, 1986, 519-524 
[7] E.J. Fordham, L.D. Hall, T.S. Ramakrishnan, M.R. Sharpe, C. Hill, Saturation Gradients in Drainage of Porous Media: NMR Imaging Measurements, A.I.Ch.E. JI. 39 (9) (1993) 1431-1443.

[8] S.M. Huang, C.G. Xie, R. Thorn, D. Snowden, M.S. Beck, Design of Sensor Electronics for Electrical Capacitance Tomography, IEE Proc. G1 139 (1992) 83-88.
[9] D. Toye, P. Marchot, M. Crine, E. L'Homme, The Use of Large Scale Computer Assisted Tomography for the Study of Hydrodynamics in Trickling Filters, Chem. Eng. Sci. 49 (1994) 5271-5280.

[10] P.J. Holden, M. Wang, R. Mann, F.J. Dickin, R.B. Edwards, Imaging Stirred-vessel Macromixing Using Electrical Resistance Tomography, A.I.Ch.E. JI. 44 (4) (1998) 780-790. 